\title{
A Randomized Controlled Trial of Vildagliptin Versus Alogliptin: Effective Switch From Sitagliptin in Patients With Type 2 Diabetes
}

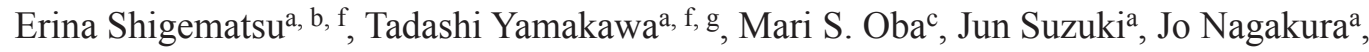 \\ Kazuaki Kadonosono ${ }^{\mathrm{d}}$, and Yasuo Terauchi ${ }^{\mathrm{e}}$
}

\begin{abstract}
Background: We investigated the effects of vildagliptin or alogliptin on blood glucose and hemoglobin A1c (HbA1c) in patients with type 2 diabetes inadequately controlled by sitagliptin.

Methods: In a single-center open-label trial, 35 patients with inadequate glycemic control on sitagliptin therapy (50 mg once daily) were randomly switched to treatment with vildagliptin (50 mg twice daily) or alogliptin ( $25 \mathrm{mg}$ once daily). After 12 weeks, patients who failed to achieve the target $\mathrm{HbA} 1 \mathrm{c}$ level of $<7.0 \%$ with vildagliptin or alogliptin treatment were switched to high-dose sitagliptin (100 mg once daily) and the effect on glycemic control was assessed.

Results: Vildagliptin did not significantly alter the mean plasma glucose level $(175.5 \pm 54.4 \mathrm{mg} / \mathrm{dL}$ vs. $179.1 \pm 73.4 \mathrm{mg} / \mathrm{dL})$ or $\mathrm{HbA} 1 \mathrm{c}$ $(8.01 \%$ vs. $8.02 \%)$ after 12 weeks. With alogliptin, mean plasma glucose increased from $175.4 \pm 50.9 \mathrm{mg} / \mathrm{dL}$ to $195.3 \pm 55.0 \mathrm{mg} / \mathrm{dL}$ after 12 weeks and $\mathrm{HbA} 1 \mathrm{c}$ increased significantly from $8.0 \%$ to $8.3 \%$ (P $<0.05)$. At 12 weeks after switching from vildagliptin to high-dose sitagliptin (100 mg daily), HbAlc was increased to $8.3 \%$, but it was significantly $(\mathrm{P}<0.05)$ reduced to the baseline level of $8.0 \%$ after switching from alogliptin. The reduction of HbAlc was significantly greater in the vildagliptin group than the alogliptin group $(\mathrm{P}=0.008)$, but the response rate (achieving the target $\mathrm{HbAlc}<7.0 \%$ ) did not differ significantly between the two groups.
\end{abstract}

Manuscript accepted for publication April 18, 2017

${ }^{a}$ Department of Endocrinology and Diabetes, Yokohama Medical Center, 4-57 Urafunecho, Minamiku, Yokohama 232-0024, Japan

${ }^{b}$ Department of Endocrinology and Metabolism, Yokohama Medical Center, 3-60-2, Harajuku, Totukaku, Yokohama 234-8575, Japan

'Department of Medical Statistics Faculty of Medicine, Toho University, 5-2116 Oomorinishi, Ootaku, Tokyo 143-0015, Japan

${ }^{\mathrm{d} D e p a r t m e n t}$ of Ophthalmology, Yokohama City University Medical Center, 4-57 Urafunecho, Minamiku, Yokohama 232-0024, Japan

eDepartment of Endocrinology and Metabolism, Yokohama City University School of Medicine, 3-9 Fukuura, Kanazawaku, Yokohama 236-0004, Japan ${ }^{\mathrm{f}}$ These authors contributed equally to this work.

gCorresponding Author: Tadashi Yamakawa, Department of Endocrinology and Diabetes, Yokohama City University Medical Center, 4-57 Urafunecho, Minami-ku, Yokohama 232-0024, Japan.

Email: yamakat@yokohama-cu.ac.jp

doi: https://doi.org/10.14740/jocmr3012w
Conclusion: The glucose-lowering effects of these three dipeptidyl peptidase-4 (DPP-4) inhibitors (vildagliptin, alogliptin, and sitagliptin) were different, and the effects of vildagliptin and sitagliptin were stronger than that of alogliptin.

Keywords: DPP-4 inhibitor; Vildagliptin; Alogliptin

\section{Introduction}

Good glycemic control is considered to be one of the cornerstones in the management of type 2 diabetes. In addition to lifestyle modification, most patients need pharmacological treatment, and most international guidelines recommend metformin as first-line therapy [1-3]. Metformin remains the optimal antidiabetic drug for initial monotherapy due to its low cost, proven safety record, weight neutrality, and possible improvement of cardiovascular outcomes [4]. The Japan Diabetes Society guideline does not make any specific recommendations about first-line therapy [5], but states that appropriate drugs should be selected according to the characteristics of each patient. Insulin sensitizers such as metformin or thiazolidinedione derivatives are preferred for obese patients, while insulin secretagogues such as sulfonylureas or glinides are recommended for non-obese patients. Recently, dipeptidyl peptidase-4 (DPP-4) inhibitors have been recommended irrespective of body mass index (BMI) because of a good safety profile, low frequency of hypoglycemia, and ease of use in elderly patients.

Among several new treatments introduced over the past few years, the new class of oral "incretin" drugs known as DPP-4 inhibitors is the most notable. DDP-4 inhibitors reduce the blood glucose level by inactivating DPP-4, an enzyme that metabolizes glucagon-like peptide-1 (GLP-1), which is a gastrointestinal hormone that augments insulin release in response to a rise of blood glucose. Sitagliptin was the first DPP-4 inhibitor, being marketed in the United States in 2007 and in Japan in 2009. Sitagliptin was followed by vildagliptin and alogliptin, but it remains most widely used in Japan and worldwide among these DPP-4 inhibitors. If a patient's HbAlc is $\geq 7 \%$ despite administration of sitagliptin, we should move to the next step of treatment, but the optimum next step is unclear. Switching from sitagliptin to another DPP-4 inhibitor, such as 
vildagliptin or alogliptin, might be one option.

Direct comparison of the effects of vildagliptin and alogliptin on glycemic control has not yet been performed in Japanese patients. Therefore, we examined the effects of vildagliptin (50 mg twice daily) and alogliptin (20 mg twice daily) on the plasma glucose level and $\mathrm{HbA} 1 \mathrm{c}$ in patients with type 2 diabetes who had inadequate glycemic control despite treatment with the usual dose of sitagliptin (50 mg daily). In addition, patients who could not achieve the target HbA1c level of $<7.0 \%$ with vildagliptin or alogliptin treatment were switched to high-dose sitagliptin therapy (100 mg daily) and the effect on glycemic control was determined.

\section{Subjects and Methods}

\section{Subjects}

Patients with type 2 diabetes attending Yokohama City University Medical Center were enrolled in this study. The inclusion criteria were men and women aged from 20 to 85 years who showed inadequate glycemic control (HbA1c from $7 \%$ to $9.5 \%$ ) on treatment with sitagliptin (50 mg daily). The exclusion criteria were as follows: 1) a history of diabetic ketoacidosis or diabetic coma within 6 months prior to study entry, 2) a history of cardiac failure, 3) surgery scheduled during the observation period of this study, 4) severe infection or severe trauma, 5) pregnant or breast-feeding female patients, 6) renal insufficiency (serum creatinine $>132.6 \mu \mathrm{mol} / \mathrm{L}$ or estimated glomerular filtration rate (e-GFR) $<30 \mathrm{~mL} / \mathrm{min}$ ), 7) severe liver dysfunction, 8) insulin therapy, 9) a history of a hypersensitivity to exenatide or liraglutide, and 10) other patients who were judged to be inappropriate for this study by the attending physician.

\section{Study protocol}

This was a single-center randomized clinical trial. Recruitment and follow-up were performed from December 2011 to April 2013 according to the above-mentioned criteria. Patients were randomly assigned (1:1) to either the alogliptin group or the vildagliptin group with stratification by baseline $\mathrm{HbA1c}(<$ $7.5 \%$ vs. $\geq 7.5 \%$ ), age ( $<60$ years vs. $\geq 60$ years $)$, and sex. Nineteen patients were assigned to the vildagliptin group and 16 to the alogliptin group. Their treatment was switched from sitagliptin (50 mg once daily) to alogliptin at $25 \mathrm{mg}$ once daily in the alogliptin group or vildagliptin at $50 \mathrm{mg}$ twice daily in the vildagliptin group. If the target $\mathrm{HbA} 1 \mathrm{c}$ level of $<7 \%$ was not achieved after 3 months, alogliptin or vildagliptin was switched to high-dose sitagliptin (100 mg daily). If $\mathrm{HbAlc}$ was $<7 \%$, the same treatment was continued for another 3 months. At 3 and 6 months after randomization, we determined the HbA1c level, plasma glucose level, and response rate (RR; the percentage of patients achieving the target $\mathrm{HbA} 1 \mathrm{c}<7.0 \%$ or plasma glucose $<130 \mathrm{mg} / \mathrm{dL}$ ).

Blood samples were collected from an antecubital vein before starting treatment and every 4 weeks during the study.
HbA1c was measured by high-performance liquid chromatography and the plasma glucose level was measured by the glucose oxidase method.

This study was approved by the Ethics Committee of Yokohama City University School of Medicine (Yokohama, Japan) and its affiliated hospital. All of the patients provided written informed consent. This study was registered with UMIN (UMIN-000010445).

\section{Statistical analysis}

We calculated the required sample size with the standard formula for a one-sided, one-sample Chi-square test and found that a sample of 29 patients would provide $80 \%$ power to reject the null hypothesis (a control rate of $20 \%$, at 3 months), assuming a control rate of $40 \%$. Considering possible loss to followup, the target sample size was set at 35 patients per group. Baseline characteristics were summarized as frequencies for categorical variables and as the mean and standard deviation (mean $\pm \mathrm{SD}$ ) for continuous variables. Comparisons between two categories were performed with the paired Student's $t$-test, while changes in continuous variables were examined by using repeated measures ANOVA and/or the Wilcoxon signed-rank test. Comparisons between groups were performed by using the $t$-test, Chi-square test, or Fisher's exact test. Imputation was not performed for missing data. The level of significance was set as $\mathrm{P}<0.05$ (two-sided). All statistical analyses were performed with SPSS version 22.0 for Windows (IBM Corporation, NY, USA).

\section{Results}

A total of 35 patients were randomized, with 19 patients assigned to the vildagliptin group and 16 patients to the alogliptin group, while 15 and 13 patients completed the study, respectively (Fig. 1). There were no significant between-group differences of baseline patient characteristics, including the age, sex, BMI, HbA1c, and renal function (Table 1). Treatment with vildagliptin for 12 weeks reduced mean $\mathrm{HbA} 1 \mathrm{c}$, although not significantly $(8.012 \%$ vs. $8.02 \%, \mathrm{P}=0.7)$. In the $15 \mathrm{sub}-$ jects from the vildagliptin group whose HbA1c was not $<7 \%$, treatment was switched from vildagliptin to high-dose sitagliptin (100 mg daily), but HbA1c was slightly higher at 24 weeks $(8.3 \%)$. During treatment with alogliptin for 12 weeks, mean $\mathrm{HbA} 1 \mathrm{c}$ increased significantly $(8.0 \%$ vs. $8.3 \%, \mathrm{P}<0.05)$. After the 13 subjects from the alogliptin group whose HbA1c was not $<7 \%$ were switched to high-dose sitagliptin, HbA1c returned to the baseline level of $8.0 \%$, showing a significant decrease $(\mathrm{P}<0.05)$. Comparison between the vildagliptin and alogliptin groups showed that the reduction of $\mathrm{HbA1c}$ was significantly greater with vildagliptin $(\mathrm{P}=0.008)$.

Changes in plasma glucose were consistent with those of HbAlc. Vildagliptin treatment did not cause a significant change in the plasma glucose level from baseline to 12 weeks $(175.5 \pm 54.4 \mathrm{mg} / \mathrm{dL}$ vs. $179.1 \pm 73.4 \mathrm{mg} / \mathrm{dL})$. After switching to high-dose sitagliptin for 12 weeks, there was also no 


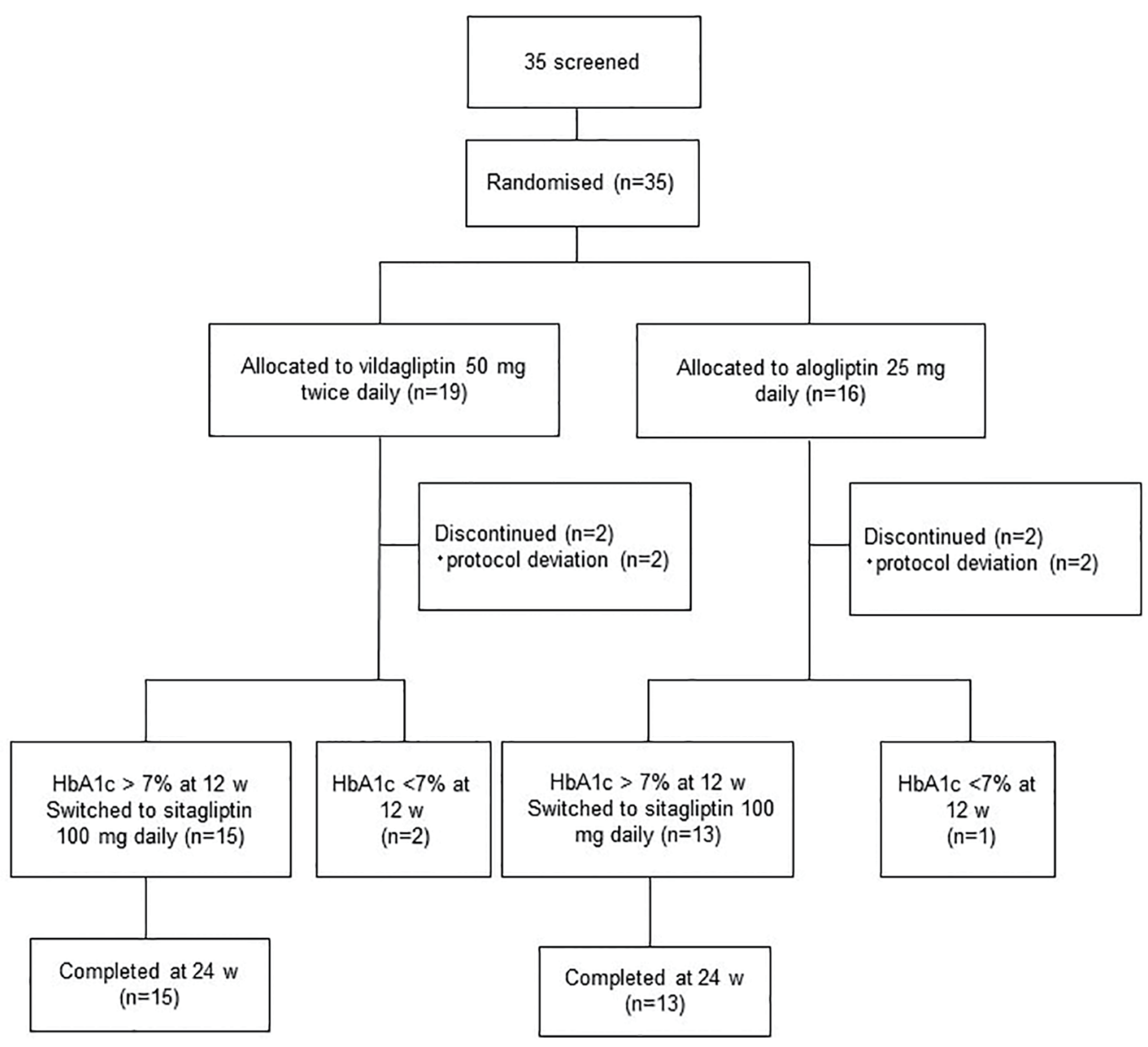

Figure 1. Assessment, randomization, and follow-up of the study patients.

significant change in plasma glucose. In the alogliptin group, the plasma glucose level increased from $175.4 \pm 50.9 \mathrm{mg} / \mathrm{dL}$ to $195.3 \pm 55.0 \mathrm{mg} / \mathrm{dL}$ after 12 weeks, but this change was not significant. After switching from alogliptin to high-dose sitagliptin treatment for 12 weeks, plasma glucose improved to $189.3 \pm 32.4 \mathrm{mg} / \mathrm{dL}$. Comparison between the vildagliptin and alogliptin groups showed no significant difference in plasma glucose levels at 12 weeks $(\mathrm{P}=0.937)$. Body weight was unchanged during the observation period and showed no significant difference between the two groups.

The RR for achieving the target HbA1c level $<7.0 \%$ at 12 weeks was not significantly different between the alogliptin

Table 1. Clinical and Laboratory Characteristics at Baseline, 12 Weeks, and 24 Weeks of Treatment With Vildagliptin or Alogliptin

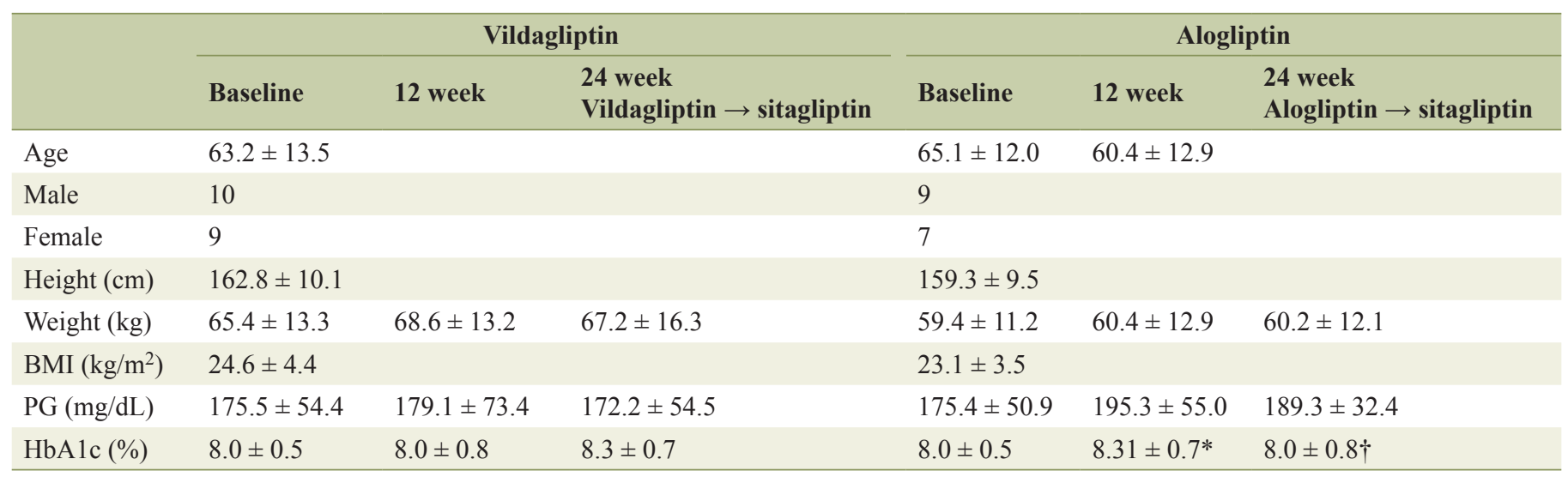

${ }^{*} P=0.05$, data compared with baseline values using the $t$-test. $\dagger P=0.05$, data compared with 12 weeks values using the $t$-test. 
Table 2. The Response Rate (RR) of Achieving the Goal Proportion of Patients Who Achieved Target Glycemic Control

\begin{tabular}{|c|c|c|c|}
\hline & Alogliptin $(\mathrm{N}=16)$ & Vildagliptin $(\mathbf{N}=19)$ & P value \\
\hline Proportion of patients who achieved $\mathrm{HbA} 1 \mathrm{c}<7.0 \%$ at 12 weeks & $1(6.3 \%)$ & $2(10.5 \%)$ & 1.000 \\
\hline Proportion of patients who achieved $\mathrm{PG}<130 \mathrm{mg} / \mathrm{dL}$ at 12 weeks & $1(6.3)$ & $7(36.8 \%)$ & 0.047 \\
\hline Proportion of patients who achieved HbA1c $<7.0 \%$ at 24 weeks & $3(9.1 \%)$ & & \\
\hline Proportion of patients who achieved $\mathrm{PG}<130 \mathrm{mg} / \mathrm{dL}$ at 24 weeks & $1(3.0 \%)$ & & \\
\hline
\end{tabular}

and vildagliptin groups, but the RR for achieving the target plasma glucose level $<130 \mathrm{mg} / \mathrm{dL}$ at 12 weeks was significantly higher in the vildagliptin group $(\mathrm{P}=0.047)$ (Table 2$)$.

\section{Discussion}

The present study was the first to directly compare efficacy between sitagliptin and two other DPP-4 inhibitors (vildagliptin and alogliptin) in patients with type 2 diabetes. There was no significant change in $\mathrm{HbAlc}$ after switching from sitagliptin to vildagliptin. On the other hand, there was a significant increase in HbA1c after switching from sitagliptin to alogliptin, while HbA1c returned to baseline when patients subsequently switched to high-dose sitagliptin. In addition, the RR for achieving the target $\mathrm{HbA} 1 \mathrm{c}<7.0 \%$ at 12 weeks was not significantly different between the vildagliptin and alogliptin groups, but the RR for achieving the target plasma glucose $<$ $130 \mathrm{mg} / \mathrm{dL}$ was significantly higher in the vildagliptin group. These results suggest that the glucose-lowering effect of vildagliptin was significantly stronger than that of alogliptin.

A meta-analysis has shown that the efficacy of DPP-4 inhibitors for reducing $\mathrm{HbAlc}$ was higher in Asian patients with type 2 diabetes than in other ethnic groups and it seems that BMI differences across ethnic groups may influence the relative HbA1c-lowering effect of these inhibitors [6]. Therefore, DPP-4 inhibitors are often selected as first-line therapy in Japan. Among the available DPP-4 inhibitors, sitagliptin is most widely used in Japan as well as elsewhere in the world. When a patient's $\mathrm{HbAlc}$ remains around $7 \%$ or higher despite treatment with sitagliptin at $50 \mathrm{mg} /$ day, the next step is usually to start combination therapy. If the glucose-lowering effect of different DPP-4 inhibitors varies, switching from sitagliptin to another DPP-4 inhibitor could also be an option. However, direct comparison of the glucose-lowering effect of DPP-4 inhibitors has not been performed before. Messori et al compared the therapeutic equivalence of five DPP-4 inhibitors by developing a standard Forest plot that incorporated information previously reported in randomized trials and reported that (in combination with metformin) linagliptin, saxagliptin, sitagliptin, and vildagliptin showed an equivalent effect, whereas alogliptin did not satisfy the equivalence criterion [7].

Direct comparison of the effects of vildagliptin and alogliptin on glycemic control has not been performed before in Japanese patients. In the present study, patients were switched from sitagliptin to vildagliptin or alogliptin, and $\mathrm{HbA} 1 \mathrm{c}$ at 12 weeks was significantly lower in the vildagliptin group than in the alogliptin group. Switching from sitagliptin to vildagliptin and subsequently switching to high-dose sitagliptin had no significant effect on HbA1c. In contrast, HbA1c increased after switching from sitagliptin to alogliptin, but then returned to baseline after subsequently switching to high-dose sitagliptin. These results suggest that the glucose-lowering effects of sitagliptin and vildagliptin might be similar and that both drugs have a significantly greater hypoglycemic effect than alogliptin, which are compatible with the results of previous studies performed in Japan [8-10].

There have been a few studies comparing the effects of different DPP-4 inhibitors. A marked increase in GLP-1 and suppression of glucagon secretion were observed in the interprandial period when patients were switched from twice daily vildagliptin treatment to sitagliptin at $100 \mathrm{mg}$ once daily $(\mathrm{P}<$ $0.01)$ [11]. It was also reported that an increase in BMI contributes to attenuation of the HbA1c-lowering effect of sitagliptin, but not vildagliptin, in Japanese type 2 diabetic patients [12]. In the present study, the BMI of the subjects was not so high, which might be the reason why the HbAlc-lowering effect of sitagliptin (50 mg/day) and vildagliptin (100 mg/day) showed no significant difference.

In vitro data about the DPP-4 inhibitory activity of the three drugs used in this study have not been consistent [1315]. We found that the glucose-lowering effect of alogliptin was slightly weaker than that of sitagliptin or vildagliptin. It has been reported that sitagliptin dose-dependently inhibits plasma DPP-4 activity over $24 \mathrm{~h}$, increases the levels of active GLP-1 and GIP, increases the insulin/C-peptide ratio, decreases the glucagon level, and reduces glycemic excursion after the OGTT [16]. Vildagliptin was reported to maintain a high GLP-1 level and suppress glucagon for over $14 \mathrm{~h}$ [17]. However, it is not clear how effectively (for how long and to what extent) alogliptin suppresses glucagon, although alogliptin has strong DPP-4 inhibitory activity and maintains a high GLP-1 concentration. Thus, it is possible that the suppressive effect of alogliptin on glucagon may be weaker or shorter compared with sitagliptin or vildagliptin.

This study showed that the glucose-lowering effects of the three DPP-4 inhibitors were slightly different. However, switching from one DPP-4 inhibitor to another, especially from sitagliptin to alogliptin, might not be a useful treatment option because the RR for achieving the target $\mathrm{HbA} 1 \mathrm{c}<7.0 \%$ was very low. In addition, increasing the dose of sitagliptin from 50 to $100 \mathrm{mg}$ did not seem to be useful, in agreement with a previous report [18]. Therefore, if the target $\mathrm{HbAlc}$ is not reached by treatment with a DPP-4 inhibitor, adding a different oral hypoglycemic drug, GLP-1 agonist, or insulin might be the next step to take. 


\section{Limitations}

There were some limitations of this study that should be noted. First, the sample size was small and the study period was short. Second, the relative efficacy of sitagliptin (50 mg daily) and vildagliptin (100 $\mathrm{mg}$ daily) could not be determined, as this study was not powered to assess small differences in efficacy. Third, changes in the serum levels of GLP-1 and glucagon were not examined.

\section{Conclusions}

The glucose-lowering effects of three DPP-4 inhibitors (vildagliptin, alogliptin, and sitagliptin) were different, with the effects of vildagliptin and sitagliptin apparently being significantly stronger than that of alogliptin.

\section{Acknowledgments}

We appreciate Mrs. Yamagiwa and Seki for secretarial assistance. We thank all the physicians who participated in this study.

\section{Conflicts of Interest}

Yasuo Terauchi received honoraria for lectures from MSD K.K.; Ono Pharmaceutical Co., Ltd; Nippon Boehringer Ingelheim Co., Ltd; Novartis Pharma K.K.; Takeda Pharmaceutical Co., Ltd; Mitsubishi Tanabe Pharma Corp.; Daiichi Sankyo Co., Ltd; Sanwa Kagaku Kenkyusho Co., Ltd; Kowa Pharmaceutical Co., Ltd; Novo Nordisk Pharma Ltd; Eli Lilly Japan K.K.; Sanofi K.K.; Shionogi \& Co., Ltd; Bayer Yakuhin, Ltd; and AstraZeneca K.K. and obtained research support from MSD K.K.; Ono Pharmaceutical Co., Ltd; Nippon Boehringer Ingelheim Co., Ltd; Novartis Pharma K.K.; Takeda Pharmaceutical Co., Ltd; Mitsubishi Tanabe Pharma Corp.; Daiichi Sankyo Co., Ltd; Sanwa Kagaku Kenkyusho Co., Ltd; Novo Nordisk Pharma Ltd; Eli Lilly Japan K.K.; Sanofi K.K.; Dainippon Sumitomo Pharma Co., Ltd; Shionogi \& Co., Ltd; Bayer Yakuhin, Ltd; Astellas Pharma, Inc.; Pfizer Japan, Inc.; and AstraZeneca K.K. Tadashi Yamakawa received honoraria for lectures from MSD K.K.; Kowa Pharmaceutical Co., Ltd; Novo Nordisk Pharma Ltd and obtained research support from AstraZeneca K.K. Jun Suzuki, Jo Nagakura, Erina Shigematsu, Kazuaki Kadonosono declare that they have no conflicts of interest.

\section{Grant Support}

None.

\section{References}

1. Nathan DM, Buse JB, Davidson MB, Heine RJ, Holman RR, Sherwin R, Zinman B. Management of hyperglyce- mia in type 2 diabetes: A consensus algorithm for the initiation and adjustment of therapy: a consensus statement from the American Diabetes Association and the European Association for the Study of Diabetes. Diabetes Care. 2006;29(8):1963-1972.

2. Nathan DM, Buse JB, Davidson MB, Ferrannini E, Holman RR, Sherwin R, Zinman B. Management of hyperglycaemia in type 2 diabetes mellitus: a consensus algorithm for the initiation and adjustment of therapy. Update regarding the thiazolidinediones. Diabetologia. 2008;51(1):8-11.

3. Inzucchi SE, Bergenstal RM, Buse JB, Diamant M, Ferrannini E, Nauck M, Peters AL, et al. Management of hyperglycemia in type 2 diabetes: a patient-centered approach: position statement of the American Diabetes Association (ADA) and the European Association for the Study of Diabetes (EASD). Diabetes Care. 2012;35(6):1364-1379.

4. Inzucchi SE, Bergenstal RM, Buse JB, Diamant M, Ferrannini E, Nauck M, Peters AL, et al. Management of hyperglycemia in type 2 diabetes, 2015: a patient-centered approach: update to a position statement of the American Diabetes Association and the European Association for the Study of Diabetes. Diabetes Care. 2015;38(1):140149.

5. Treatment Guide for Diabetes 2014 - 2015. Japan Diabetes Society: Bunkodo, 2014.

6. Kim YG, Hahn S, Oh TJ, Kwak SH, Park KS, Cho YM. Differences in the glucose-lowering efficacy of dipeptidyl peptidase-4 inhibitors between Asians and non-Asians: a systematic review and meta-analysis. Diabetologia. 2013;56(4):696-708.

7. Messori A, Fadda V, Maratea D, Trippoli S, Marinai C. Testing the therapeutic equivalence of alogliptin, linagliptin, saxagliptin, sitagliptin or vildagliptin as monotherapy or in combination with metformin in patients with type 2 diabetes. Diabetes Ther. 2014;5(1):341-344.

8. Yoshimasa Y. The Effect of novel DPP-4 inhibitor alogliptin monotherapy, combination therapy, switch from other DDP-4 inhibitor therapy in patients with type 2 diabetes of inadequate glycemic control. Progress in Medicine. 2011;31:1791-1804.

9. Koide D, Yamazaki T. Meta-analysis of DPP-4 inhibitor: Effect of sitagliptin and alogliptin to reduce HbA1c. Progress in Medicine. 2010;30:2645-2651.

10. Murakami I. Effects of bidirectional switching in Sitagliptin, Vildagliptin and alogliptin on Glucose Control in Type 2 Diabetes Mellitus. Therapeutic Research. 2014;35:305-311.

11. Marfella R, Barbieri M, Grella R, Rizzo MR, Nicoletti GF, Paolisso G. Effects of vildagliptin twice daily vs. sitagliptin once daily on 24-hour acute glucose fluctuations. J Diabetes Complications. 2010;24(2):79-83.

12. Bando Y, Kanehara H, Aoki K, Hisada A, Toya D, Tanaka $\mathrm{N}$. Obesity may attenuate the HbAlc-lowering effect of sitagliptin in Japanese type 2 diabetic patients. J Diabetes Investig. 2012;3(2):170-174.

13. Croxtall JD, Keam SJ. Vildagliptin: a review of its use in the management of type 2 diabetes mellitus. Drugs. 2008;68(16):2387-2409. 
14. Gallwitz B. Review of sitagliptin phosphate: a novel treatment for type 2 diabetes. Vasc Health Risk Manag. 2007;3(2):203-210.

15. Thomas L, Eckhardt M, Langkopf E, Tadayyon M, Himmelsbach F, Mark M. (R)-8-(3-amino-piperidin-1-yl)7-but-2-ynyl-3-methyl-1-(4-methyl-quinazolin-2-ylm ethyl)-3,7-dihydro-purine-2,6-dione (BI 1356), a novel xanthine-based dipeptidyl peptidase 4 inhibitor, has a superior potency and longer duration of action compared with other dipeptidyl peptidase-4 inhibitors. J Pharmacol Exp Ther. 2008;325(1):175-182.

16. Herman GA, Bergman A, Stevens C, Kotey P, Yi B, Zhao $\mathrm{P}$, Dietrich B, et al. Effect of single oral doses of sitagliptin, a dipeptidyl peptidase-4 inhibitor, on incretin and plasma glucose levels after an oral glucose tolerance test in patients with type 2 diabetes. J Clin Endocrinol Metab. 2006;91(11):4612-4619.

17. Balas B, Baig MR, Watson C, Dunning BE, LiguerosSaylan M, Wang Y, He YL, et al. The dipeptidyl peptidase IV inhibitor vildagliptin suppresses endogenous glucose production and enhances islet function after single-dose administration in type 2 diabetic patients. J Clin Endocrinol Metab. 2007;92(4):1249-1255.

18. Iwamoto Y, Taniguchi T, Nonaka K, Okamoto T, Okuyama K, Arjona Ferreira JC, Amatruda J. Dose-ranging efficacy of sitagliptin, a dipeptidyl peptidase-4 inhibitor, in Japanese patients with type 2 diabetes mellitus. Endocr J. 2010;57(5):383-394. 